\title{
Hadron Spectroscopy with Photons at CLAS and CLAS12
}

\section{A. Filippi*}

I.N.F.N. sez. di Torino

E-mail: filippi@to.infn.it

\begin{abstract}
An overview of the most recent hadron spectroscopy results by the CLAS experiment at JLAB, obtained by exploiting a real photon beam, is reported. For the first time the experiment could operate with a beam of suitable intensity and energy resolution to allow complete studies of light mesons and baryons dynamics to be performed. An upgraded version of the apparatus, CLAS12, which recently started taking data at the new CEBAF machine (that is providing an electron beam of about twice the previous energy), will soon be able to deliver new results based on the interactions of a virtual photon beam. The potentialities of the new CLAS12 apparatus for hadron spectroscopy studies are also described.
\end{abstract}

XIII Quark Confinement and the Hadron Spectrum - Confinement2018

31 July - 6 August 2018

Maynooth University, Ireland

${ }^{*}$ Speaker. 


\section{Light Hadron Spectroscopy as a tool to understand QCD}

Hadron spectroscopy is one of the most effective tools to study QCD confinement, on account of its connection to the mechanism of mass generation. In fact, most of the non-perturbative effects which are relevant at low energies (or, equivalently, large distances) are still largely unknown and only a phenomenological study of the properties of hadrons and the features of their mass spectrum can help in the understanding the rôle of gluons and the origin of confinement.

Spectroscopy studies at low energies involving light quarks only are particularly sensitive probes of the confinement features; in this energy range, chiral symmetry breaking effects in the non-perturbative regime can also be involved. On the other hand, the interaction between heavy quarks is useful to probe the strong force mainly at small distances and can benefit from the application of non-relativistic perturbative techniques, that are providing fairly good descriptions of the processes.

$\mathrm{QCD}$, as a non-Abelian group theory, allows for the existence of mesonic and baryonic structures composed by gluons only, or by mixtures of gluons and quarks. The search for such new states, which may exhibit quantum numbers not allowed by the ordinary $q \bar{q}$ or $q q q$ structures, has been one of the goals of spectroscopy studies in the last fifty years, but so far no conclusive evidence has been provided for them. However, quite recently a remarkable step forward was made in the Lattice QCD (LQCD) computational studies, which are now able to reproduce with good precision a relevant part of the known particles spectrum, and offer predictive power about where the lightest exotic structures are expected to be found. The lightest exotic structures in the meson spectrum, of hybrid $q \bar{q} g$ composition, are expected to have a mass of about $1600 \mathrm{MeV}$ and $2 \mathrm{GeV}$, with $1^{-+}$and $2^{+-}$quantum numbers, respectively $[1,2]$. This is indeed the mass region which will be investigated by the new generation of experiments at the CEBAF electron machine. On the other hand, concerning the baryon spectrum, LQCD is able to reproduce satisfactorily the spectrum description provided by the non-relativistic quark model based on the $\mathrm{SU}(6) \times \mathrm{O}(3)$ symmetry and also to predict a number of new excitations; some of them were indeed observed in the last years mostly in electroproduction reactions [3].

LQCD is also able to predict the location of the lightest hybrid baryons of the spectrum, characterized by a $q^{3} g$ structure but featuring the same quantum numbers as ordinary baryons. The lowest mass hybrids are expected to form a cluster of $N^{*}$-like states with $J^{P}=1 / 2^{+}$around 2.2 $\mathrm{GeV}$, about $1.3 \mathrm{GeV}$ above the mass of ordinary nucleons [4]. Since there is no possibility to disentangle them from ordinary excitations just on the basis of quantum numbers, their identification must be determined from other properties, like the $Q^{2}$ dependence of the transition form factors; this technique was already exploited for the characterization of the Roper resonance [5], as will be described in the following.

Whereas in the last decade a wealth of results, especially on baryon dynamics, was extracted by CLAS in the study of electron induced reactions (and a selection of them will be described in the following), the search for both meson and baryon exotics is a central goal of the latest generation experiments running at the new CEBAF machine: indeed, this topic plays a major part of the approved scientific program of the new CLAS12 experiment $[6,7]$. 


\section{Hadron spectroscopy in photon induced interactions}

Photoproduction reactions offer complementary features as compared to those induced by hadronic probes $[8,9,10]$. First of all, in this case the interaction amplitudes may be evaluated perturbatively with high precision, based of well-known QED diagrams. Photons can be real or virtual: the latter are exchanged in electron induced reactons, and their energy (and virtuality) can be determined by the measurement of the momentum and direction of the scattered electron measured in the final state.

In reactions induced by photons the probability of producing spin 1 particles in the final state is large as no spin flip is needed, which is not the case with a pion or kaon beam; this feature can be fruitfully exploited, for instance, in the search of a spin 1 exotic meson, which could in principle be produced at rates comparable to those of ordinary mesons. Last but not least, the production of quarkonia, and in particular $s \bar{s}$ excitations which at the moment are still poorly known, is expected to occur at larger rates in reactions induced by a vector probe.

\subsection{Photoproduction experiments with real and virtual photons at the CEBAF machine in JLAB}

The CEBAF machine at JLAB was devised to provide a continuous electron beam of high intensity and unprecedented resolution to several experiments located in three experimental halls. In the first part of its operation the maximum electron energy reached $6 \mathrm{GeV}$; CLAS (CEBAF Large Acceptance Spectrometer) was the main experiment dedicated to hadron spectroscopy studies, in particular to the study of reactions induced by photons that could be produced by brehmsstrahlung in a dedicated part of the experimental setup. CLAS, whose description is given elsewhere [11], operated at the CEBAF electron machine up to 2012, when it was shut down to upgrade it to a twice as large energy. The new machine was completed by 2016 and nowadays it is fully operational, delivering an electron beam with energy up to $12 \mathrm{GeV}$. A new experimental hall was built, that hosts now the main experiment at JLAB dedicated to hadron spectroscopy studies, GlueX [12]. While GlueX exploits the same technique used by CLAS for the production of a real photon beam, the upgraded version on the CLAS detector, CLAS12, cannot run with real photons beams. This is due to the dimensions of the experimental hall where the experiment is located, which are not sufficient to steer the non-interacting $11 \mathrm{GeV}$ electron beam away. For this reason, the hadron spectroscopy program proposed for CLAS12 is based on electron interactions with the exchange of virtual photons which can be tagged if the forward scattered electron is measured with precision.

As far as hadron spectroscopy studies are concerned, GlueEx and CLAS12 present complementary features. While GluEx is a $4 \pi$ spectrometer with uniform acceptance and good hermeticity, its particle identification performances, at least in the original setup, are limited compared to CLAS12, which features a better momentum resolution at the expense of a reduced acceptance due to its more compact dimensions. A thorough description of the CLAS12 apparatus may be found in Ref. [13].

When the electron scattering occurs in CLAS12 at very low transferred momentum $\left(Q^{2}<10^{-1}\right.$ $\mathrm{GeV}^{2}$ ) the electrons in the final state are produced at very small polar angles. A dedicated part of the CLAS12 apparatus, the Forward Tagger, was devised to measure these electrons. For momentum transfers in the interval $0.01<Q^{2}<0.3 \mathrm{GeV}^{2}$, the electrons scattered with a polar angle 
between $2.5^{\circ}$ and $4.5^{\circ}$ in the lab have an energy in the (0.5-4.5) GeV range, being produced by the interaction of (6.5-10.5) GeV quasi-real photons on protons. Measuring the electron momentum, each virtual photon can be tagged. The use of the CLAS12 spectrometer to measure in coincidence the particles produced in the photoproduction reaction will allow a complete event recontruction to be performed, necessary for meson spectroscopy studies. With this tagging technique one can measure also the virtual photon polarization, that is linear and can be deduced, event by event, from the energy and the angle of the scattered electron. The systematic uncertainty affecting the polarization depends only on the electron momentum resolution. High electron currents may be used, and therefore a good luminosity can be obtained even with thin targets, that are not operable with real photon bremsstrahlung beams. For instance, using a $5 \mathrm{~cm}$ long $\mathrm{LH}_{2}$ target, the resulting hadronic rate will be equivalent to that achievable by a real photon flux of about $5 \times 10^{8} \mathrm{r} / \mathrm{s}$.

The Forward Tagger equipment, described in detail in Ref. [14], is located about $190 \mathrm{~cm}$ away from the target and fits within a $5^{\circ}$ cone around the beam axis. It is made up of:

- an electromagnetic calorimeter (FT-Cal): composed by $332 \mathrm{PbWO}_{4}$ crystals, $20 \mathrm{~cm}$ long and with square $15 \times 15 \mathrm{~mm}^{2}$ cross-section. It is used to identify the scattered electron and measure its energy, from which the photon energy and its polarization can be deduced (the polarization being given by $\varepsilon^{-1} \sim 1+v^{2} /\left(E E^{\prime}\right)$, where $v=E-E^{\prime}$ is the photon energy, and $E$ and $E^{\prime}$ the energies of the incident and of the scattered electron, respectively). It is also used to provide a fast trigger signal. Its expected design resolution is $\sigma_{E} / E \sim(2 \% / \sqrt{E(\mathrm{GeV})} \oplus 1 \%)$;

- a scintillator hodoscope made of plastic scintillator tiles: located in front of the calorimeter, is used to veto photons; its spatial and timing resolution is required to be comparable with FT-Cal's;

- a tracker: located in front of the hodoscope and composed of Micromegas detectors, is used to measure the angle of the scattered electron and the photon polarization plane.

A wide hadron spectroscopy program based on photon induced interactions was proposed at CLAS12, both for the study of meson and hadron spectra. Research items are for instance the search for exotics and hybrids, the study of hyperon excitations (of nucleon resonances, $\Delta$ 's or strange hyperons), the study of light meson decay patterns and the search of pentaquarks. CLAS12 started taking data at the end of 2017 and is now currently extracting the first physics outputs which are very promising in term of resolutions and particle identification efficiency, both for charged and for neutral particles.

\section{Meson spectroscopy with real photons: some selected results from CLAS}

\subsection{The scalar glueball search case}

In spite of the efforts by the experiments in the early Nineties, in particular those studying antinucleon annihilations, like Crystal Barrel (see for instance [15, 16, 17] and references therein) and OBELIX (see $[18,19,20,21]$ and references therein), the full composition of the scalar meson sector is not completely clear yet. Observations have been made of several structures whose 
existence was not foreseen by the Constituent Quark Model; one of them, the $f_{0}(1500)$, seemed to have the right features to be identified as the lightest scalar glueball of the mesonic spectrum $[15,16,17,18,19,20]$. Among these, the most important one is the fact that the structure was observed to decay in several channels, a clear hint to its flavor-blindness. However, more data are still desirable to confirm these properties as several other structures could tentatively be identified as scalars as well. All the existing observations make the interpretation of the scalar sector difficult, since it is populated by more broad and overlapping states than expected. In this mass region one should also recall the existence of the $\sigma$ state (also known as $f_{0}(600)$ ), corresponding to a very broad $\pi \pi$ non-resonant iso-scalar $S$-wave interaction, whose nature and properties are still unclear. Also scarcely known are the features of the $\kappa$, analogous to $\sigma$, observed in the $K \bar{K}$ channel.

A search was carried on in CLAS exploiting the $\gamma p \rightarrow p K_{S}^{0} K_{S}^{0}$ reaction [22], with real photons of energy in the $(2.7-3)$ and $(3.1-5.1) \mathrm{GeV}$ ranges. The $K_{S}^{0}$ were fully reconstructed through their decay to two pions, while the proton was identified from the event missing mass. A strong correlation between the two $K_{S}^{0}$ was found, which allowed a clean sample of good statistics to be collected, enough to perform selections in momentum transfer $t\left(=Q^{2}\right)$. The selection in $|t|$ is useful to understand the production mechanism of the intermediate states: small values of momentum transfers are correlated to a dominant production from the $t$-channel, while $s$-channel production is characterized by a wider range of transferred momenta. Fig. 1 shows the distributions of the $\left(K_{S}^{0} K_{S}^{0}\right)$ invariant mass system, after proper background subtraction, in two momentum transfer ranges: for $|t|<1 \mathrm{GeV}^{2}$ on the left side, and for $|t|>1 \mathrm{GeV}^{2}$ on the right. A clean peak at about $1500 \mathrm{MeV}$ appears in the first case, while at larger momentum transfer no evidence for it can be observed. This means that the structure at $1500 \mathrm{MeV}$ is predominantly produced via $t$-channel, and this could be a hint to a possible interpretation as glueball of the $f_{0}(1500)$ observed in its $K_{S}^{0} K_{S}^{0}$ decay mode.
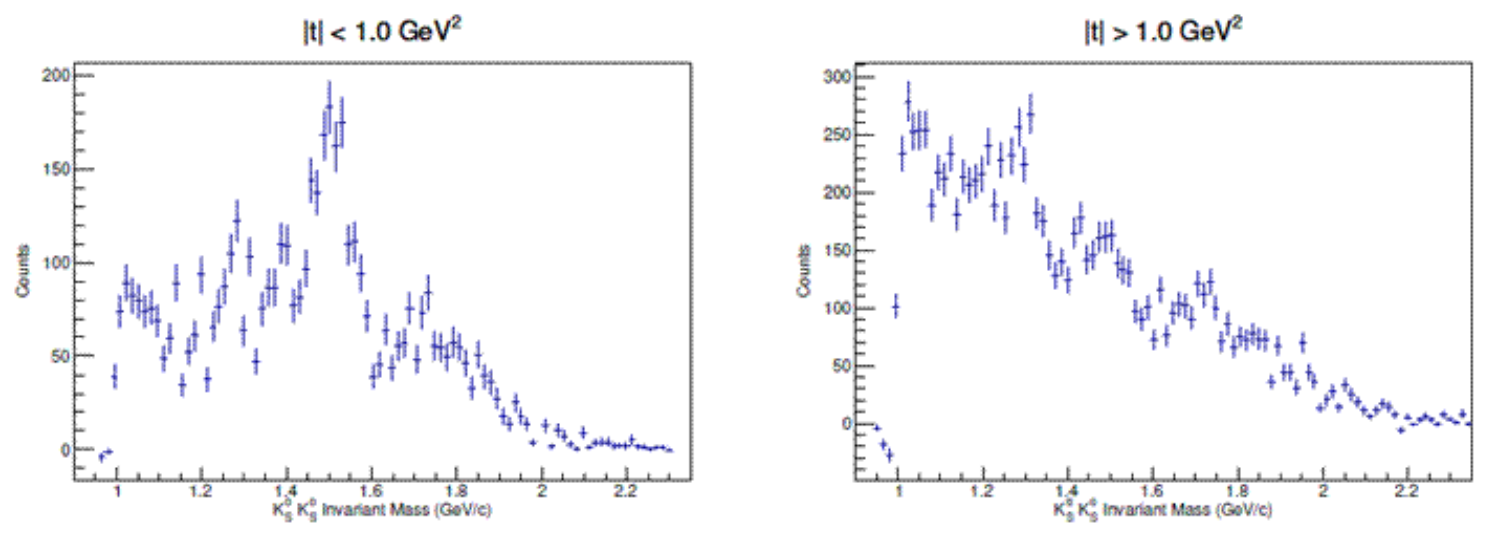

Figure 1: Invariant mass of the $\left(K_{S}^{0} K_{S}^{0}\right)$ system for events selected in the $\gamma p \rightarrow p K_{S}^{0} K_{S}^{0}$ reaction, in two momentum transfer ranges: left, for $|t|<1 \mathrm{GeV}^{2}$, right for $|t|>1 \mathrm{GeV}^{2}$.

In order to fully characterize the features of this state a complete spin-parity analysis of the sample is required. Unfortunately, the task is not straightforward due to the limited apparatus acceptance at small forward and backwards angles. Nonetheless, an angular analysis was attempted to test which of the spin-parity hypotheses for the events in the peak region would provide a better 
fit to the Gottried-Jackson angular distributions: the scalar hypothesis for the observed resonance is mostly supported, and the identification as the $f_{0}(1500)$ looks more likely.

\subsection{The axial/pseudoscalar sector at $1.4 \mathrm{GeV}$ case}

Kaonia radial excitations were widely studied in the past together with pseudoscalar $(\eta)$ excitations in the same mass range to search for possible exotic states. Many observation of $\eta$ 's and $f_{1}$ mesons have been reported since the Sixties, and the issue of the overlap of many axial and pseudoscalar states and the difficulty of their identification has been discussed, posing the so-called $E / \imath$ puzzle [21]. While $N \bar{N}$ annihilation experiments, and in particular OBELIX, could provide a solution to this puzzle, addressing the production of several pseudoscalar and axial states, high statistics photoproduction reactions are now expected to deliver new complementary information which will be able to improve the knowledge in this sector.

A systematic study was performed by CLAS to study the photoproduction of states, decaying to $\eta \pi^{+} \pi^{-}$and $K^{0} K^{ \pm} \pi^{\mp}$ and recoiling against a proton, in the $\gamma p$ reaction with photons in the $(3-3.8) \mathrm{GeV}$ energy range [23]. Fig. 2 shows the missing mass plot of the system recoiling against a proton for $\gamma p \rightarrow p \eta \pi^{+} \pi^{-}$selected events, where a clean signal due to the $\eta^{\prime}(958)$ appears, together with a structure at about $1280 \mathrm{MeV}$, that can tentatively be identified as the $f_{1}(1285)$.

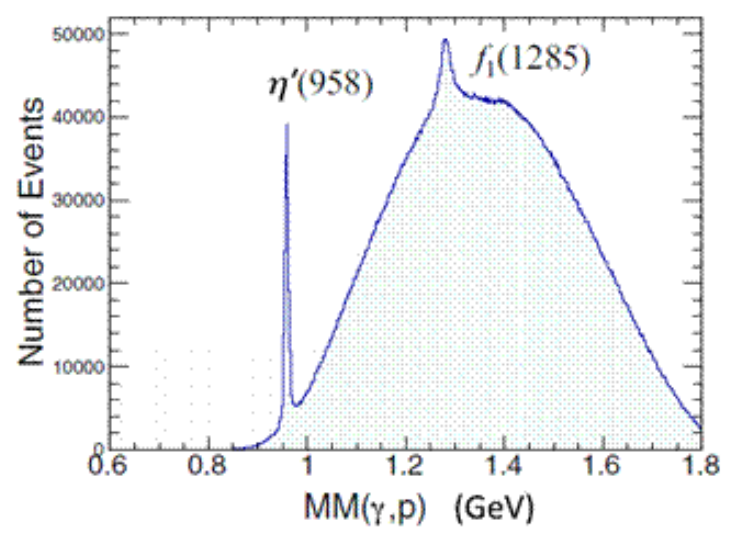

Figure 2: Missing mass recoling against a proton for events selected in the $\gamma p \rightarrow p \eta \pi^{+} \pi^{-}$reaction.

Concerning the reaction with kaons, the $K^{ \pm}$were identified by CLAS through time of flight techniques, while the $K^{0}$ via the missing mass information. The missing mass plots for the system recoiling against the proton for events selected in the two channels $\gamma p \rightarrow p \overline{K^{0}} K^{+} \pi^{-}$and $\gamma p \rightarrow$ $p K^{0} K^{-} \pi^{+}$are shown, respectively, in Fig. 3a) and b).

In both of them a clear peak appears at about $1.3 \mathrm{GeV}$, but no further evidence for higher mass states that could be attributed to additional pseudoscalar structures, like as the $\eta(1405)$ and $\eta(1470)$, or to axial states, such as the $f_{1}(1420)$ of $f_{1}(1510)$, is present.

From the richest $\eta \pi^{+} \pi^{-}$sample, one can get information on the identification of the observed state. There are a few hints that support the identification as axial $f_{1}(1285)$ against the pseudoscalar $\eta(1295)$. The first one is given by the values obtained for the mass and width of the signal, $M=$ $(1281.0 \pm 0.8) \mathrm{MeV}$ and $\Gamma=(18.4 \pm 1.4) \mathrm{MeV}$, which are closer to those already observed for $f_{1}(1285)$ in other reactions [24]. The ratio itself of the decay rates in $\eta \pi^{+} \pi^{-}$versus $K \bar{K} \pi$, that 

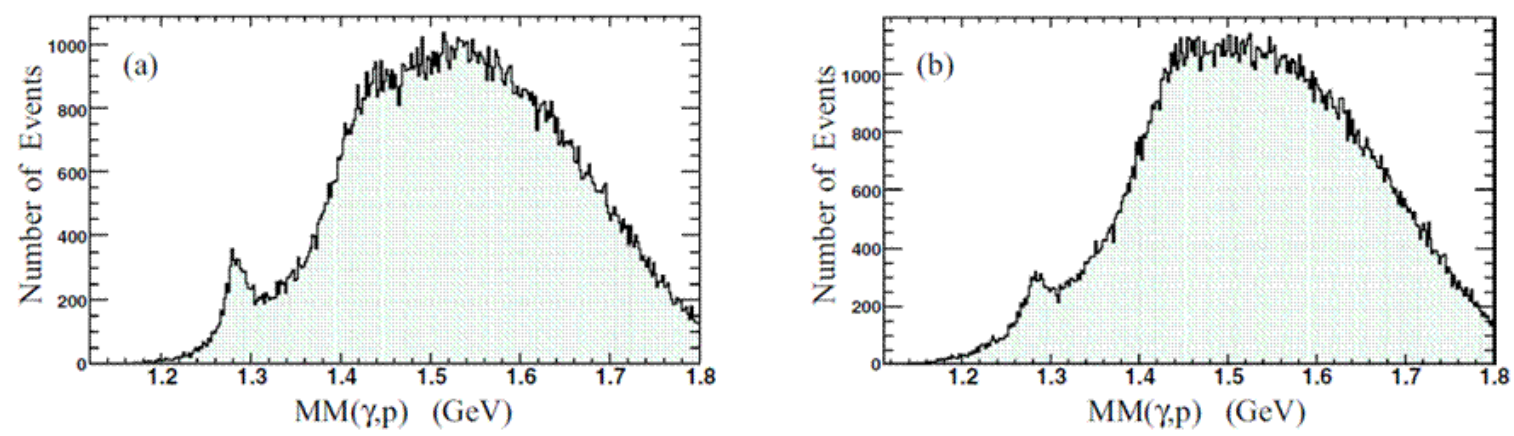

Figure 3: Missing mass recoling against a proton for events selected in the a) $\gamma p \rightarrow p \overline{K^{0}} K^{+} \pi^{-}$and b) $\gamma p \rightarrow p K^{0} K^{-} \pi^{+}$reactions.

amounts to about five, is consistent with the value quoted by PDG for the $f_{1}(1285)$ decays, while no such ratio was ever measured for $\eta(1295)$ [24].

The second hint is given by the trend of the differential cross section, for events selected in the $\eta^{\prime}(958)$ band and around $1280 \mathrm{MeV}$ : the two are remarkably different as a function of the center-of-mass angle, indicating a different production mechanism and possibly a different spin configuration of the produced state.

\section{Meson spectroscopy with virtual photons: future prospects with CLAS12}

In order to perform a reliable partial-wave analysis a good number of selected events, on the order of at least a few thousand per mass or momentum transfer bins, are necessary. Studies with Monte Carlo data have been made to check the capability of CLAS12 to provide a good enough data sample to extract reliable information on new possible physical effects. To this purpose, some benchmark reactions have been investigated, in which new signals in known conditions have been injected (i.e., with known spin and fixed production rates), to check the reproducibility of the simulated spectrum against acceptance distortions and an approximate amplitude description.

An example of a tested reaction is $\gamma p \rightarrow \pi^{+} \pi^{+} \pi^{-} n$, in which a sum of eight isobar channels was considered, corresponding to the known resonant states in $S-, P-, D-, F$-waves decaying in $\rho \pi$ or $f_{2}(1270) \pi$, plus an exotic signal in $P$-wave (standing for the $\pi_{1}(1600) 1^{-+}$state in its $\rho \pi$ decay). The simulated data were folded with the CLAS12 acceptance, processed through the full reconstruction chain, and then fitted via a proper partial-wave set in the framework of the isobar model and in selected bins of transferred momentum $t$. The obtained results, in two $t$ bins marked by points of different colors, are shown in Fig. 4. All known resonances $-a_{2}(1320)$ in $D$-wave, $a_{1}(1260)$ in $D$-wave, $\pi_{2}(1670)$ in the two decay channels $\rho \pi$ ( $P$ - and $F$-wave) and $f_{2} \pi$ ( $S$ - and $D$-wave) - are correctly reproduced at the right mass values, as well as the additional exotic signal. Its injected weight, at the level of $2 \%$ (corresponding to a production cross section of about 200 $\mathrm{nb})$, was reproduced correctly as well. 

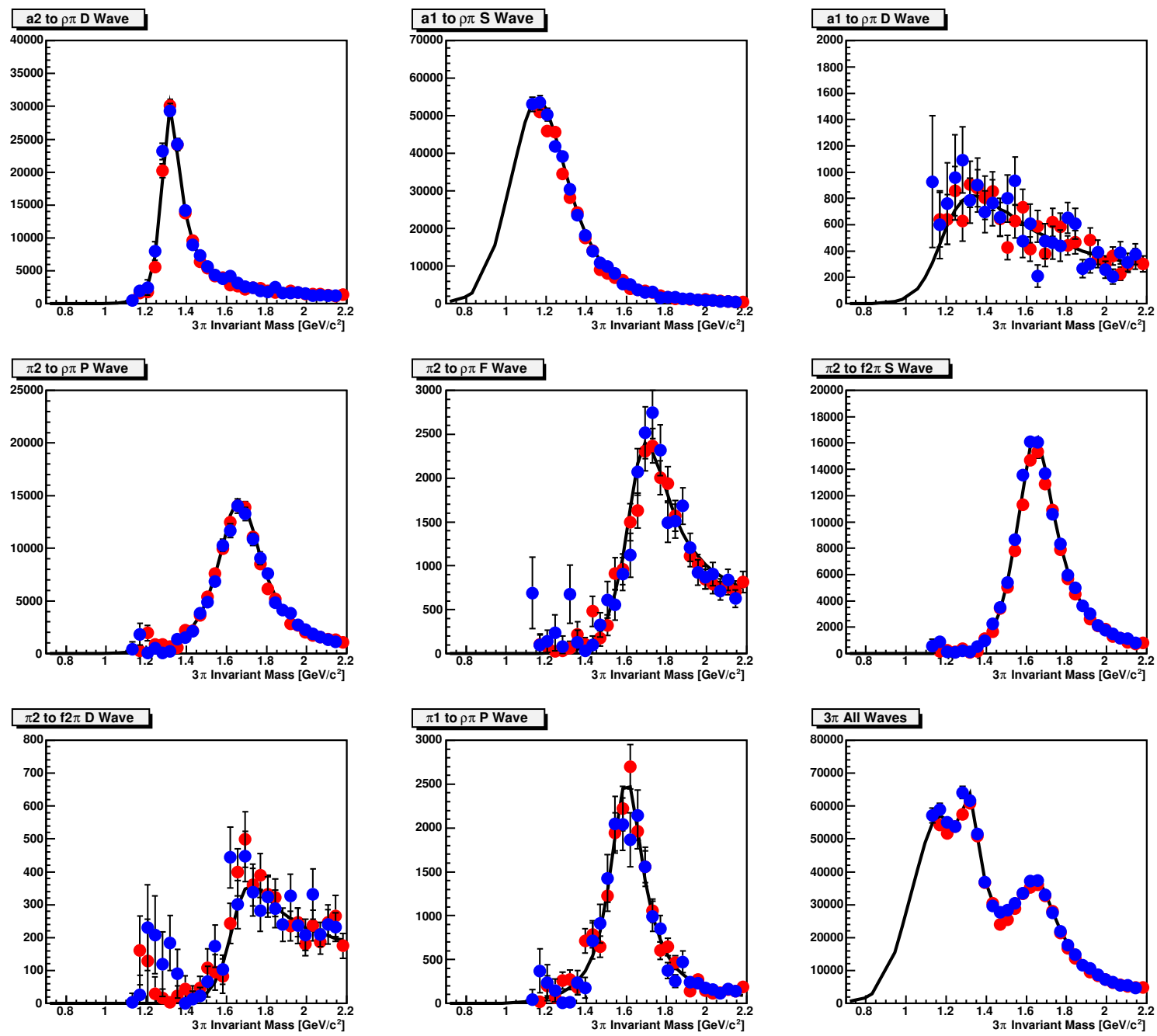

Figure 4: Simulations of intensities of eight isobar channels in the $\gamma p \rightarrow \pi^{+} \pi^{+} \pi^{-} n$ channel, filtered through CLAS12 acceptance. In the bottom row, the last pad on the right corresponds to the total $3 \pi$ intensity, while the pad in the center correspond to the exotic signal injected in the pseudo-data sample. The black lines are the generated partial waves, while the points are the fit results for pseudo-data selected in the two bins centered at $t=0.2 \mathrm{GeV}^{2}\left(0.25 \mathrm{MeV}^{2}\right.$ wide, blue points) and $t=0.5 \mathrm{GeV}^{2}\left(1 \mathrm{MeV}^{2}\right.$ wide, red points).

\section{Baryon spectroscopy with real photons: some selected results from CLAS}

\subsection{Study of new baryon resonances}

The study of baryon resonances received an unprecedented boost when electroproduction high precision data, mainly collected by CLAS, started to be available. In the latest years many new states, predicted by LQCD calculations, were indeed found and they updated the scenario sketched in 2010 , mostly based on low statistics results provided by $\pi N$ scattering reactions and pion photoproduction.

With more high quality data improved models could be tested to perform complete PWA's, like the Bonn-Gatchina multichannel model [25] which provided a more precise description of the 
reaction dynamics. Moreover, precious additional information could be provided by the study of polarization variables. In particular, in a study of the $E$ asymmetry in the $\gamma p \rightarrow \pi^{+} n$ reaction, the application of the Bonn-Gatchina model provided a largely improved description of the data, especially in the lower energy bins, as compared to previous models (SAID [26], Jülich-Bonn [27]) which did not include the contributions from new baryonic excitations [28].

Fig. 5 shows, in the upper panel, how the experimental data from CLAS compare to the earlier analyses based on the cited models not including the mentioned data-sets, and, in the lower panel, how the results improve once the new data are included in the analyses.

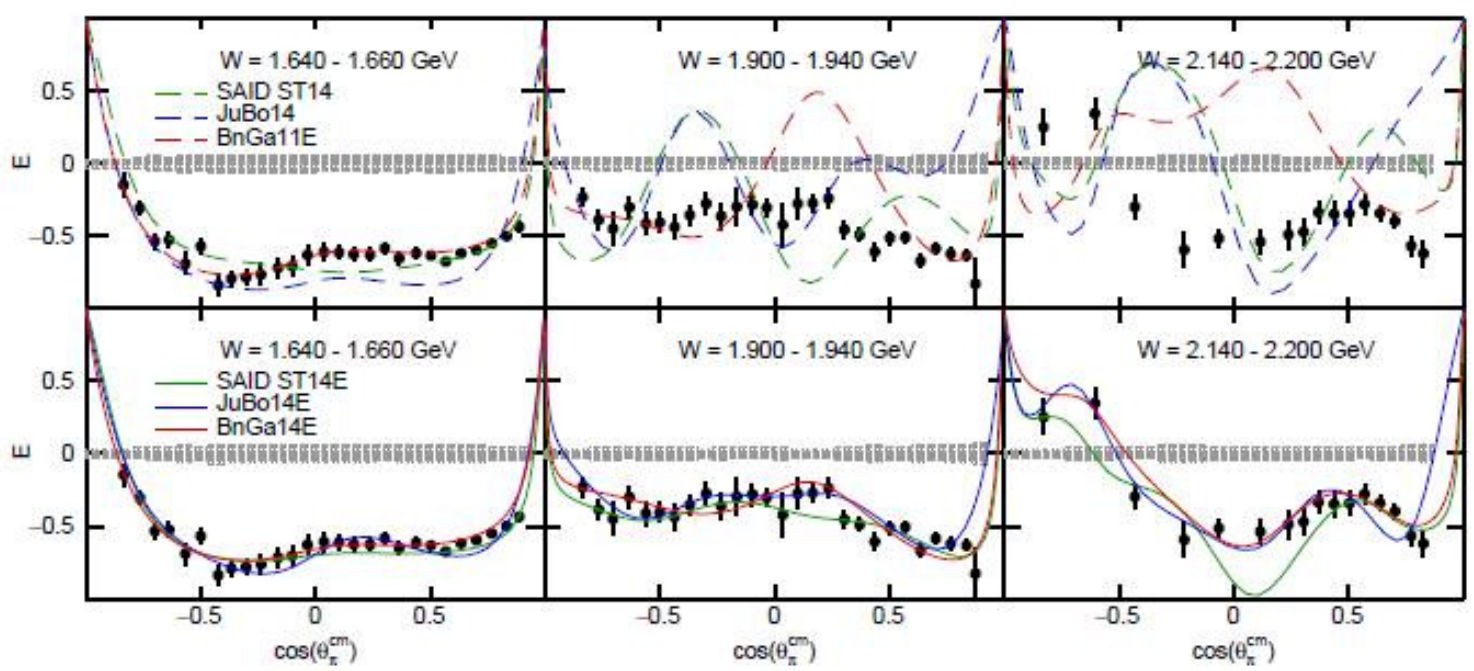

Figure 5: Beam-target polarization observable $E$ measured by CLAS in the $\gamma p \rightarrow \pi^{+} n n$ reaction in selected energy bins. The shaded bands indicate systematic uncertainties; the dashed curves in the upper panels are results from the SAID ST14 [26], Jülich14-Bonn [27], and BnGa11E [25] analyses. The solid curves in the lower panels are results from updated analyses including the new CLAS E data. From ref. [28].

\subsection{Search for parity doublets}

The observation of both meson and baryon parity doublets, featuring the same spin, about the same mass and opposite parities suggested the possibility that chiral symmetry could be restored in highly excited resonances. In particular, a striking recurrence of $\Delta^{*}$ doublets of similar mass occurs for $J^{P}=1 / 2^{ \pm}, 3 / 2^{ \pm}$and $5 / 2^{ \pm}$. However, the $7 / 2^{ \pm}$doublet, studied applying the Bonn-Gatchina model to the combined data samples collected by CLAS and CBELSA/TAPS in the photoproduced $\pi^{0} p, \pi^{+} n, K \Sigma, \pi^{0} \pi^{0} p$ and $\pi^{0} \eta p$ final states disproves this hypothesis [29]. In fact, while the $\left(7 / 2^{+}\right) \Delta$ state appears in all channels at about $1950 \mathrm{MeV}$, the $(7 / 2)^{-} \Delta$ resonance lies at $2180 \mathrm{MeV}$ (and can be identified with the $\Delta(2200)$ ), in contrast to what could be expected in the hypothesis of chiral symmetry restoration, also with respect to the observed width and decay modes.

\section{$5.3 Q^{2}$ evolution of the transition form factor and the Roper resonance}

The availability of a large high precision data sample allows the study of hadron structure evolution at different wavelengths $\left(Q^{2}\right)$, offering an alternative tool to infer the properties of hadron 
production. In particular, the $Q^{2}$ dependence of the helicity amplitudes (also known as transition form factors) can help rule out some hypotheses on the nature of the data in mass regions where possible new signals are observed. This is the case in the study of the $N(1440) 1 / 2^{+}$, the so-called Roper resonance, for which a possible identification as a hybrid baryon was suggested.

Were this the case, one would expect, on the basis of the hybrid model, a steep drop with $Q^{2}$ of the transverse helicity amplitude $A_{1 / 2}$, and a suppressed longitudinal amplitude $S_{1 / 2}$. The experimental findings, based on CLAS $n \pi^{+}$and $p \pi^{+} \pi^{-}$data in the $0 \leq Q^{2} \leq 4.5 \mathrm{GeV}^{2}$ range, show some inconsistency with this hypothesis, with a different behaviour of the two helicity amplitudes compared to the expectations [30,31]. In particular, the $A_{1 / 2}$ amplitude is larger than expected by the model and changes sign, while for $S_{1 / 2}$ one has a decreasing trend far from being zero as suggested. Especially at low $Q^{2}$ the discrepancy with the expectations from the non relativistic Quark Model is wide. This suggests as more likely the possibility that the $N(1440) 1 / 2^{+}$state might be an ordinary $q^{3}$ radial excitation, interplaying rather sizeably with the outer meson-baryon cloud.

Fig. 6 shows the trend of the $A_{1 / 2}$ and $S_{1 / 2}$ helicity amplitudes for CLAS data: note how the experimental data compare with model expectations (dashed curve: Dyson-Schwinger equation (DSE)/QCD calculations [32]; solid curve: Light Front relativistic Quark Model (LFRQM) [33] with momentum dependent quark mass) in the hypothesis of the existence of a hybrid baryonic structure in the $1440 \mathrm{MeV}$ region.
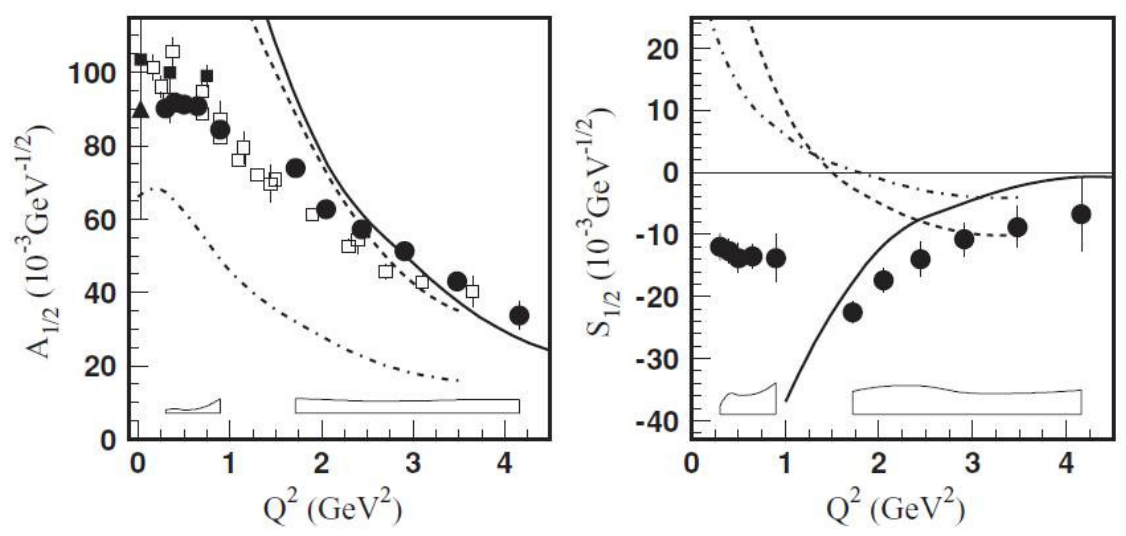

Figure 6: The electrocoupling amplitudes $A_{1 / 2}$ and $S_{1 / 2}$ as a function of the transferred momentum $Q^{2}$ for data in the Roper resonance $N(1440)$ mass region, data from CLAS [30]. The dashed curve is from the DSE/QCD calculation [32], the solid curve is from LFRQM with momentum-dependent quark mass [33].

\subsection{Study of $\Xi$ baryons and excitations}

The study of $\Xi$ and its radial excitations in photoproduction reactions started with CLAS with the analysis of the inclusive $\gamma p \rightarrow K^{+} K^{-} X$ and $\gamma p \rightarrow K^{+} K^{+} \pi^{-} X$ final states. In spite of the limited statistics clean signals for the production of the $\Xi(1320)$ and $\Xi(1530)$, in a roughly 10:1 ratio, were observed [34]. Other investigations were done on a sample with photon energy in the (3.5 - 5.4) $\mathrm{GeV}$ range, to assess the total cross section for the $\Xi$ production. Even though the only 
significant emerging signal is the $\Xi(1530)$, upper limits for the production of the $\Xi(1630), \Xi(1690)$ and $\Xi(1820)$ radial excitations, at the maximum level of $1.09 \mathrm{nb}$ for the latter, were extracted [35].

A large statistics study of such states is part of the "Very Strange Baryon" program proposed at CLAS12 [36].

\section{Search for new hybrids and multi-strange baryons at CLAS12}

A proposal was approved to study the production of hybrid baryons at CLAS12. The purpose is to investigate in detail the nuclear and $\Delta$ high mass excitations and possibly find signatures of $q^{3} g$ structures, whose identification is problematic as they share the same decay channels with ordinary baryons and do not exhibit exotic quantum numbers. The expected statistics will allow an analysis of the $Q^{2}$ dependence of helicity amplitudes which, as in the case previously described for the Roper resonance, will help identifying the nature of the possible signatures which are expected to occur around $1800 \mathrm{MeV}$ [7].

As mentioned in Section 5.4, part of the baryon spectroscopy CLAS12 program is dedicated to the refinement of the studies of the properties of the $\Xi$ baryon and its radial excitations, including its polarization and helicity asymmetries, as well as of other still unknown baryons with more units of strangeness, like the $\Omega$ 's. The main purpose is to shed light on the strangeness onset in photoproduction reactions, which has necessarily to be excited from the quark sea. To successfully pursue such a program, the required experimental setup foresees the inclusion of the Forward Tagger which is essential in order to allow the detection of the $\gamma p \rightarrow K^{+} K^{-} p, K^{+} \Lambda \pi^{0}$ and $K^{+} K^{+} \Xi^{-}$ reactions. In 80 days of data taking at half nominal value magnetic field, 7-8 thousands $\Omega^{-}$baryons are expected to be collected at CLAS12, and at least one order of magnitude more of the rarest $\Xi$ excitations [36].

\section{Summary and conclusions}

Many problems are still open on both meson and baryon spectroscopy studies, for both the observation and identification of several structures still waiting for confirmation. At JLAB, the new CEBAF accelerator is nowadays able to provide high intensity, linearly polarized photon beams (both with real and low $Q^{2}$-tagged virtual photons). These characteristics, combined with high performance detectors featuring excellent particle identification performance and momentum resolution, will allow CLAS12 to collect abundant and high quality data. These will be the basis for robust Partial Wave analyses, whose framework had been elaborated and tuned over the years training on the statistics collected by the first generation CLAS experiment. First class quality data and results are expected soon, complementary to the achievements in hadron spectroscopy pursued by other current high energy physics experiments such as BESIII, LHCb, COMPASS and BelleII.

\section{References}

[1] J. Dudek et al., Phys. Rev. $D 82$ (2010), 034508

[2] J. Dudek et al., Phys. Rev. D83 (2011), 111502

[3] R. G. Edwards et al., Phys. Rev. $D 84$ (2011), 074508 
[4] J.J. Dudek and R.G. Edwards Phys. Rev. D85 (2012), 054016

[5] T. Barnes and F.E. Close, Phys. Lett. B123 (1989), 89

[6] CLAS Collaboration, Meson Spectroscopy with low $Q^{2}$ electron scattering in CLAS12, Exp-11-005 "MesonEx", Proposal Submitted to Jefferson Lab PAC41

[7] CLAS Collaboration, A Search for Hybrid Baryons in Hall B with CLAS12, Exp-12-16-010, Proposal Submitted to Jefferson Lab PAC44

[8] F. Close and P. Page, Phys. Rev. D52 (1995), 1706

[9] A. Afanasev and P. Page, Phys. Rev. D57 (1998), 6771

[10] A. Szczepaniak and M. Swat, Phys. Lett. B516 (2001), 72

[11] B.A. Mecking et al., Nucl. Instr. Meth. A503 (2003), 513

[12] GlueX Collaboration, Mapping the Spectrum of Light Mesons and Gluonic Excitations with Linearly Polarized Photons, Proposal to Jefferson Laboratory PAC30, 2006

[13] CLAS Collaboration, CLAS12 Technical Design Report, Tech. Rep. Jefferson Laboratory, 2008

[14] CLAS Collaboration, CLAS12 Forward Tagger (FT) Technical Design Report, Tech. Rep. Jefferson Lab, 2012

[15] Crystal Barrel Collaboration, C. Amsler et al., Phys. Lett. 3342 (1995), 433

[16] Crystal Barrel Collaboration, C. Amsler et al., Phys. Lett. $B 353$ (1995), 571

[17] Crystal Barrel Collaboration, C. Amsler et al., Phys. Lett. $B 385$ (1996), 425

[18] OBELIX Collaboration, A. Bertin et al., Phys. Lett. B408 (1997), 476

[19] OBELIX Collaboration, A. Bertin et al., Phys. Rev D57 (1998), 55

[20] OBELIX Collaboration, M. Bargiotti et al., Phys. Lett. B561 (2003), 233

[21] A. Masoni, C. Cicalò and G.L. Usai, J. Phys. G32 (2006), R293

[22] CLAS Collaboration, S. Chandavar et al., Phys. Rev. C97 (2018), 025203

[23] CLAS Collaboration, R. Dickson et al., Phys. Rev. C93 (2016), 065202

[24] Particle Data Group, C. Patrignani et al., Chin. Phys. C40 (2016), 100001

[25] A.V. Anisovich et al., Eur. Phys. J. A48 (2012), 15

[26] R. L. Workman et al., Phys. Rev. C86 (2012), 015202

[27] D. Rönchen et al., Eur. Phys. J. A50 (2014), 101

[28] CLAS Collaboration, S. Strauch et al., Phys. Lett. B750 (2016), 53

[29] A. V. Anisovich et al., Phys. Let.. $\mathbf{B 7 6 6}$ (2017), 357

[30] CLAS Collaboration, I.G. Aznauryan et al., et al., Phys. Rev. C80 (2009), 055203

[31] CLAS Collaboration, V.I. Mokeev et al., Phys. Rev. C86 (2012), 035203

[32] J. Segovia et al., Phys. Rev. Lett 115 (2015), 171801

[33] I.G. Aznauryan and V. Burkert, Phys. Rev. C85 (2012), 055202

[34] CLAS Collaboration, L. Guo et al., Phys. Rev. C76 (20017), 205208

[35] CLAS Collaboration, J. Goetz, Phys. Rev. C98 (2018), 062201

[36] CLAS Collaboration, Photoproduction of the Very Strangest Baryons on a Proton Target in CLAS12, Exp-12-11-005a "Very Strange Experiment" 